\title{
SOME SUMS CONNECTED WITH QUADRATIC RESIDUES
}

\author{
L. CARLITZ
}

1. A well known theorem of Dirichlet asserts that if $p$ is a prime $\equiv 3(\bmod 4)$, then

$$
\sum_{r=1}^{(p-1) / 2}\left(\frac{r}{p}\right)>0
$$

that is, among the integers $1,2, \cdots,(p-1) / 2$, there are more quadratic residues of $p$ than nonresidues. A concise proof of this theorem has recently been given by Moser [2]; Whiteman [4] has proved several closely related results.

In the present note we indicate a generalization of (1.1) and in particular that for $p \equiv 3(\bmod 4)$,

$$
\begin{aligned}
& (-1)^{k+1} \sum_{k=1}^{(p-1) / 2}\left(\frac{h}{p}\right) B_{2 k+1}\left(\frac{h}{p}\right) \text { and } \\
& (-1)^{k} \sum_{h=1}^{(p-1) / 2}\left(\frac{h}{p}\right) E_{2 k}\left(\frac{2 h}{p}\right)
\end{aligned}
$$

are positive for $k \geqq 0$, while for $p \equiv 1(\bmod 4)$,

$$
\begin{gathered}
(-1)^{k+1} \sum_{h=1}^{(p-1) / 2}\left(\frac{h}{p}\right) B_{2 k}\left(\frac{h}{p}\right) \text { and } \\
(-1)^{k} \sum_{h=1}^{(p-1) / 2}\left(\frac{h}{p}\right) E_{2 k-1}\left(\frac{2 h}{p}\right)
\end{gathered}
$$

are positive for $k \geqq 1$. In (1.2) and (1.3), $B_{k}(x)$ and $E_{k}(x)$ denote the Bernoulli and Euler polynomials, respectively, of degree $k$.

2. In the familiar summation $[1$, p. 153]

$$
\sum_{r=1}^{p-1}\left(\frac{r}{p}\right) e^{2 \pi i r n / p}= \begin{cases}\left(\frac{n}{p}\right) p^{1 / 2} & (p \equiv 1(\bmod 4)), \\ i\left(\frac{n}{p}\right) p^{1 / 2} & (p \equiv 3(\bmod 4)),\end{cases}
$$

which is valid for all $n$, we first take $p \equiv 3(\bmod 4)$. Then $(2.1)$ becomes 1952.

Presented to the Society, September 5, 1952; received by the editors May 12, 


$$
\sum_{r=1}^{m}\left(\frac{r}{p}\right) \sin \frac{2 \pi r n}{p}=\frac{1}{2}\left(\frac{n}{p}\right) p^{1 / 2} \quad(p=2 m+1) .
$$

If we multiply both sides of (2.2) by $a_{n}$ and sum over $n$, then

$$
\sum_{r=1}^{m}\left(\frac{r}{p}\right) f\left(\frac{2 r}{p}\right)=\frac{1}{2} p^{1 / 2} \sum_{n=1}^{\infty}\left(\frac{n}{p}\right) a_{n}
$$

where

$$
f(x)=\sum_{n=1}^{\infty} a_{n} \sin n \pi x
$$

If we assume $a_{n}$ real and $\sum a_{n}$ absolutely convergent, then we may be able to infer from (2.3) that the sum in the left member is positive. For example let $a_{r s}=a_{r} a_{s}$ for arbitrary integers $r, s$ and let $\left|a_{n}\right|<1$ for all $n$, then

$$
\sum_{n=1}^{\infty}\left(\frac{n}{p}\right) a_{n}=\prod_{q}\left\{1-\left(\frac{q}{p}\right) a_{q}\right\}^{-1}>0 ;
$$

the product extends over all primes $q$. In some instances the assumption of absolute convergence can be weakened.

In particular if we make use of the expansion $[3$, p. 65]

$$
B_{2 k+1}(x)=(-1)^{k+1} \frac{2(2 k+1) !}{(2 \pi)^{2 k+1}} \sum_{n=1}^{\infty} \frac{\sin 2 n \pi x}{n^{2 k+1}},
$$

then (2.3) becomes

$$
\begin{aligned}
(-1)^{k+1} \sum_{h=1}^{m}\left(\frac{h}{p}\right) B_{2 k+1}\left(\frac{h}{p}\right) & \\
= & \frac{(2 k+1) ! p^{1 / 2}}{(2 \pi)^{2 k+1}} \sum_{n=1}^{\infty} \frac{\left(\frac{n}{p}\right)}{n^{2 k+1}} \\
& =\frac{(2 k+1) ! p^{1 / 2}}{(2 \pi)^{2 k+1}} \prod_{q}\left\{1-\frac{\left(\frac{q}{p}\right)}{q^{2 k+1}}\right\}^{-1},
\end{aligned}
$$

where the product extends over all primes $q$. We infer that the left member of (2.4) is positive for $k \geqq 0$ (the case $k=0$ requires special treatment since the convergence of the series on the right is not absolute).

Similarly it follows from $[3$, p. 66] 


$$
E_{2 k}(x)=(-1)^{k} \frac{4(2 k) !}{\pi^{2 k+1}} \sum_{n=0}^{\infty} \frac{\sin (2 n+1) \pi x}{(2 n+1)^{2 k+1}}
$$

that

$$
\begin{aligned}
(-1)^{k} \sum_{k=1}^{m}\left(\frac{h}{p}\right) E_{2 k}\left(\frac{2 h}{p}\right)= & \frac{2(2 k) !}{\pi^{2 k+1}} p^{1 / 2} \sum_{n=0}^{\infty} \frac{\left(\frac{2 n+1}{p}\right)}{(2 n+1)^{2 k+1}} \\
= & \frac{2(2 k) !}{\pi^{2 k+1}} p^{1 / 2} \prod_{q>2}\left\{1-\frac{\left(\frac{q}{p}\right)}{q^{2 k+1}}\right\}^{-1} .
\end{aligned}
$$

We infer that the left member of (2.5) is positive for $k \geqq 0$ (again the case $k=0$ requires special treatment; compare [2]).

3. For $p \equiv 1(\bmod 4),(2.1)$ becomes

$$
\sum_{r=1}^{m}\left(\frac{r}{p}\right) \cos \frac{2 \pi r n}{p}=\frac{1}{2}\left(\frac{n}{p}\right) p^{1 / 2} \quad(p=2 m+1),
$$

by means of which we can again assert an identity like (2.3) where $f(x)$ is now a cosine series. However we shall discuss only the particular cases corresponding to the Bernoulli and Euler polynomials. In the first place, making use of $[3$, p. 65]

$$
B_{2 k}(x)=(-1)^{k+1} \frac{2(2 k) !}{(2 \pi)^{2 k}} \sum_{n=1}^{\infty} \frac{\cos 2 n \pi x}{n^{2 k}},
$$

we get

$$
(-1)^{k+1} \sum_{h=1}^{m}\left(\frac{h}{p}\right) B_{2 k}\left(\frac{h}{p}\right)=\frac{(2 k) !}{(2 \pi)^{2 k}} p^{1 / 2} \sum_{n=1}^{\infty} \frac{\left(\frac{n}{p}\right)}{n^{2 k}} .
$$

It follows that the left member of (3.2) is positive for $k \geqq 1$.

Secondly, by means of $[3$, p. 66]

$$
E_{2 k-1}(x)=(-1)^{k} \frac{4(2 k-1) !}{\pi^{2 k}} \sum_{n=0}^{\infty} \frac{\cos (2 n+1) \pi x}{(2 n+1)^{2 k}}
$$

we infer

$$
(-1)^{k} \sum_{k=1}^{m}\left(\frac{h}{p}\right) E_{2 k}\left(\frac{2 h}{p}\right)=\frac{2(2 k-1) !}{\pi^{2 k}} \sum_{n=0}^{\infty} \frac{\left(\frac{2 n+1}{p}\right)}{(2 n+1)^{2 k}} .
$$


It follows that the left member of (3.3) is positive for $k \geqq 1$.

\section{REFERENCES}

1. E. Landau, Vorlesungen uber Zahlentheorie, Leipzig, 1927, vol. 1.

2. L. Moser, $A$ theorem on quadratic residues, Proceedings of the American Mathematical Society vol. 2 (1951) pp. 503-504.

3. N. E. Nörlund, Vorlesungen über Differenzenrechnung, Berlin, 1924.

4. A. L. Whiteman, Theorems on quadratic residues, Mathematics Magazine vol. 23 (1949) pp. 71-74.

DURE UNIVERSITY

\section{FACTORIZATION OF $n$-SOLUBLE AND n-NILPOTENT GROUPS}

\section{REINHOLD BAER}

If $n$ is an integer [positive or negative or 0], and if the elements $x$ and $y$ in the group $G$ meet the requirements

$$
(x y)^{n}=x^{n} y^{n} \text { and }(y x)^{n}=y^{n} x^{n},
$$

then we term the elements $x$ and $y n$-commutative. It is not difficult to verify that $n$-commutativity and $(1-n)$-commutativity are equivalent properties of the elements $x$ and $y$, that (-1)-commutativity implies ordinary commutativity, and that commuting elements are $n$-commutative.

From any concept and property involving the fact that certain elements [or functions of elements] commute, one may derive new concepts and properties by substituting everywhere $n$-commutativity for the requirement of plain commutativity. This general principle may be illustrated by the following examples.

$n$-abelian groups are groups $G$ such that $(x y)^{n}=x^{n} y^{n}$ for every $x$ and $y$ in $G$. They have first been discussed by F. Levi [3]; and they will play an important rôle in our discussion. Grün [2] has introduced the $n$-commutator subgroup. It is the smallest normal subgroup $J$ of $G$ such that $G / J$ is $n$-abelian; and $J$ may be generated by the totality of elements of the form $(x y)^{n}\left(x^{n} y^{n}\right)^{-1}$ with $x$ and $y$ in $G$. Dual to the $n$-commutator subgroup is the $n$-center. It is the totality of elements $z$ in $G$ such that $(z x)^{n}=z^{n} x^{n}$ and $(x z)^{n}=x^{n} z^{n}$ for every $x$ in $G$; see Baer [1] for a discussion of this concept.

Received by the editors May 1, 1952. 